\title{
Combined effect of biopriming and polymer coating on chemical constituents of root exudation in chilli (Capsicum annuum L.) cv. $\mathrm{K} 2$ seedlings
}

\author{
S. Sathya*, S. Lakshmi and S. Nakkeeran \\ Department of Seed Science and Technology, Tamil Nadu Agricultural University, Coimbatore-641001 (T.N.), INDIA \\ Department of Plant pathology, Tamil Nadu Agricultural University, Coimbatore - 641001 (T.N.), INDIA \\ *Corresponding author. E-mail: sathya.agri03@gmail.com \\ Received: march 7, 2016; Revised received: August 16, 2016; Accepted: November 16, 2016
}

\begin{abstract}
A study was carried out to analyze the different volatile compounds in bioprimed chilli (Capsicum annuum L.) seedlings of 15 and 30 day old. A common compound found in two stages of chilli seedlings was hydroxylamine, dimethoxydimethyl silane, hexadecanoic acid, 15-methyl- methyl ester. Majority of the compounds in bacterized seedlings had antimicrobial activity. The results on GCMS analysis revealed that, root exudates collected from 15 and 30 days old bacterized seedlings with $B$. amyloliquefaciens VB7 and polymer coating released more number of volatile compounds ( 65 and 20 compounds respectively) than control ( 5 and 15 compounds respectively). The root exudates of 15 day old seedling released more volatile compounds ( 65 nos) than 30 days ( 20 nos) old seedling.
\end{abstract}

Keywords: Antimicrobial activity, B. amyloliquefaciens VB7, Polymer coating, Volatile compounds

\section{INTRODUCTION}

Chilli (Capsicum annuum) is one of the important spice crop cultivated around the world for its pungency and colour. The pungency is due to the active principle capsicin content in the skin and septa of the fruit. It is also used in beverages and preparation of medicines (Zagade et al., 2012). Owing to the potentiality, intensive cultivation of chilli is attacked by several diseases leading to loss of yield in terms of quality and quantity. Among these diseases, damping off incited by Pythium spp. is responsible for 90 per cent of plant death either as pre or post-emergence in nurseries and fields (Sowmini, 1961). Pythium species are soil borne plant pathogenic fungi, which cause pre and post emergence damping off (Shah Smith and Burns, 1996).

Though fungicides offer a greater degree of protection against pathogens, accumulation of residues in the fruits and their adverse effect on beneficial soil microorganisms and the environment cannot be ignored. Therefore, biocontrol agents appear to hold promise in disease management. Since, biological control is a key component of integrated disease management; it is active against specific pathogens for wider application (Nakkeeran et al., 2006). For effective management of any soil borne disease, the introduced antagonist should colonize the roots (Weller, 1984). The successful antagonist should colonize the rhizosphere at the time of seed germination itself and the antagonist should move from spermosphere to rhizosphere and establish (Weller and Cook, 1983).

Seedling health is determined by the root health.
Biopriming and polymer coating helps in developing a strong root system by promoting biological control of plant diseases besides improving the root system for the active acquisition of water and nutrients for better quality of seedlings (Dorlodot et al., 2007). Heydecker (1973) defined seed priming as a presowing seed invigouration treatment in which seeds are soaked in osmotic solution that allows them to imbibe water and go through the first phase of germination, but does not permit radicle or plumule protrusion through seed coat. Seed treatment with biocontrol agents along with priming agents may serve as an important means of managing many of the soil and seed borne diseases, the process often known as "biopriming" (Rao et al., 2007). Polymer coating is application of a thin, uniform layer of polymer over seeds without significantly increasing seed size and weight. The film formed around the seed acts as a physical barrier, which has been reported to reduce leaching of inhibitors from the seed coverings and may restrict oxygen diffusion to the embryo (Vanangamudi et al., 2003).

Root exudation is a part of rhizodeposition process, which is a major source of soil organic carbon released by plant roots (Nguyen, 2003). The quantity and quality of root exudates are determined by plant species, age of an individual plant and external factors like biotic and abiotic stresses. Root exudation clearly represents a significant carbon cost to the plant with young seedlings typically exuding about 30-40 per cent of their fixed carbon as root exudates (Whipps, 1990). Root exudates contain released ions (i.e. $\mathrm{H}^{+}$), inorganic acids, oxygen and water, but mainly consist of carbon-based 
compounds (Bais et al., 2006).

Hydroponics is a technology for growing plants in nutrient solutions (water and fertilizers) with or without the use of an artificial medium. Hydroponic culture can significantly increase plant growth and produce uniform, stress-free root and shoot material that can be harvested throughout the life span of the plant (Gibeaut, 1997).

The aim of the study was to i) identify the volatile compounds released from the chilli seedling root exudates and compare the root exudates composition of bacterized and untreated seedlings ii) compare the root exudates composition of 15 and 30 days seedlings grown on hydroponic conditions

\section{MATERIALS AND METHODS}

Seed treatment: Chilli seeds were surface sterilized with 80 per cent ethanol for $5 \mathrm{~min}$ and rinsed four times with distilled water. The seeds were bioprimed with liquid based formulation of Bacillus amyloliquefaciens VB7 by soaking the seeds for a period of $12 \mathrm{~h}$ and later the seeds were removed and immediately coated with polymer $\left(10 \mathrm{ml} \mathrm{kg}^{-1}\right.$ of seed $)$ and then shade dried at room temperature $\left(28 \pm 2^{\circ} \mathrm{C}\right)$.

Preparation of root exudates: Seeds bioprimed with 6 per cent B. amyloliquefaciens VB7 and untreated seeds were kept for germination using paper medium (between paper). The 14 days old seedlings of uniform size were transplanted into glass test tubes containing $50 \mathrm{ml}$ Hoagland's nutrient solution (Hoagland and Arnon in 1950) prepared with deionized water. Root exudates were collected on 15 and 30 days. The collected liquid was filtrated through a column $(20 \mathrm{~mm}$ diameter) containing $100 \mathrm{ml}$ of XAD-4 resin, followed by elution with $50 \mathrm{ml}$ methanol and condensed on rotary evaporator (Model IRA ${ }^{@} \mathrm{RV} 10$ ) at $40^{\circ} \mathrm{C}$. The solution, with a total volume of $25 \mathrm{ml}$, was then refrigerated at $-20{ }^{\circ} \mathrm{C}$ until use.

Identification of root exudates: Concentrated methanol solution $(5 \mathrm{ml})$ was transferred to XAD-4 resin column with $200 \mathrm{ml} 80$ per cent ether +20 per cent acetate elution to allow the natural evaporation of methanol. The eluate was concentrated under vacuum to dryness and then dissolved in one $\mathrm{ml}$ of HPLC grade methanol (Qun et al., 2012). The main component was used in the identification of the root exudates through gas chromatography-mass spectrometry (GC-MS, GC Agilent - 7890B, MS Agilent - 5977A MSD) analysis. One $\mu \mathrm{L}$ aliquots of the reaction mixture were injected directly into the gas chromatograph, operating under the following conditions:

The initial temperature of $80^{\circ} \mathrm{C}$ was kept for one min, then raised to $250^{\circ} \mathrm{C}$ at a rate of $8^{\circ} \mathrm{C} \mathrm{min}^{-1}$, then raised to $300^{\circ} \mathrm{C}$ at a rate of $12^{\circ} \mathrm{C} \mathrm{min}^{-1}$ and held for $5 \mathrm{~min}$, total $\mathrm{GC}$ run time was $30 \mathrm{~min}$. Injector temperature was $240^{\circ} \mathrm{C}$.

\section{RESULTS AND DISCUSSION}

Identification of volatile compounds in the root exudates of 15 days old chilli seedlings: The compounds identified in root exudates of nontreated seedlings were shown in Fig.1 and Table 1. The major chemical constituents were hydroxylamine with peak area percentage $(91.98 \%)$, dimethoxydimethylsilane $(3.47 \%)$ and phenylephrine (2.68\%). Among the identified compounds 2.7 per cent had antimicrobial activity.

Sixty five chemical constituents (Fig.2 and Table 2) have been identified from biopriming with 6 per cent B. amyloliquefaciens VB7 and polymer coating @10 $\mathrm{ml}^{-1} \mathrm{~kg}$ of seed. Among the identified compounds 62.8 per cent had antimicrobial activity. The major chemical constituents with maximum peak area percentage in bacterized seedling root exudates were identified as hydroxylamine $(10.13 \%)$, n-decanoic acid $(9.24 \%), 1$ -hexadecanol (7.99\%), Z-8-Methyl-9-tetradecenoic acid (5.99\%), cis-undec-4-enal (5.26\%), 13-Octadecenal, (Z)(4.46\%), 13-Tetradecenal (4.35\%), 9-Octadecenal (3.35 $\%)$, Tetrapentacontane, 1,54-dibromo (2.93\%), transundec-4-enal (2.84 \%), 1,2-Benzenedicarboxylic acid, mono (2-ethylhexyl) ester (2.06\%).

The common compounds identified in treated and nontreated 15 day seedlings root exudates were hydroxylamine, dimethoxydimethylsilane and hexadecanoic acid -15 methyl- methyl ester.

Among the fatty acids, hexadecanoic acid known to have the antibacterial, antifungal activity (Mahadkar et al., 2013) antioxidant, nematicide, 5-alpha reductase inhibitor (Selvamangai and Anusha, 2012). Tetradecanoic acid is known to have potential antibacterial, antifungal activity (Mahadkar et al., 2013) antioxidant and nematicide (Selvamangai and Anusha, 2012). Octadecanoic acid, pentadecanoic acid and heptadecanoic acid have potential antibacterial and antifungal activity (Mahadkar et al., 2013). Another group of fatty acids with potential antifungal activity is the cyclopropane fatty acids (Carballeira, 2008). Alcohols, such as 1hexanol have antifungal activity and prevent diseases (Archibold et al., 1997).

Hydroxylamines promote seed germination by inhibition of hydrogen peroxide $\left(\mathrm{H}_{2} \mathrm{O}_{2}\right)$ decomposition by catalase (Hendricks and Taylorson, 1974). Hydroxylamine is a strong reductant and a strong chelating agent. It reacts to form oximes with aldehydes and ketones or nitrogen ethers with aldehydes, when mono-N substituted (Taylor and Baker, 1937). The marked chelating capacities of hydroxylamine for the iron atoms of haem proteins and the definite but lower capacities for $\mathrm{N}$ aliphatic substituted hydroxylamines indicate the presence of this type of action in seeds.

The use of fatty acids as antifungal agents offers some advantages. Liu et al. (2008) proposed that antifungal fatty acids can replace chemicals in use to control plant diseases worldwide, which negatively impact the envi- 
S. Sathya et al. / J. Appl. \& Nat. Sci. 8 (4): 2141-2154 (2016)

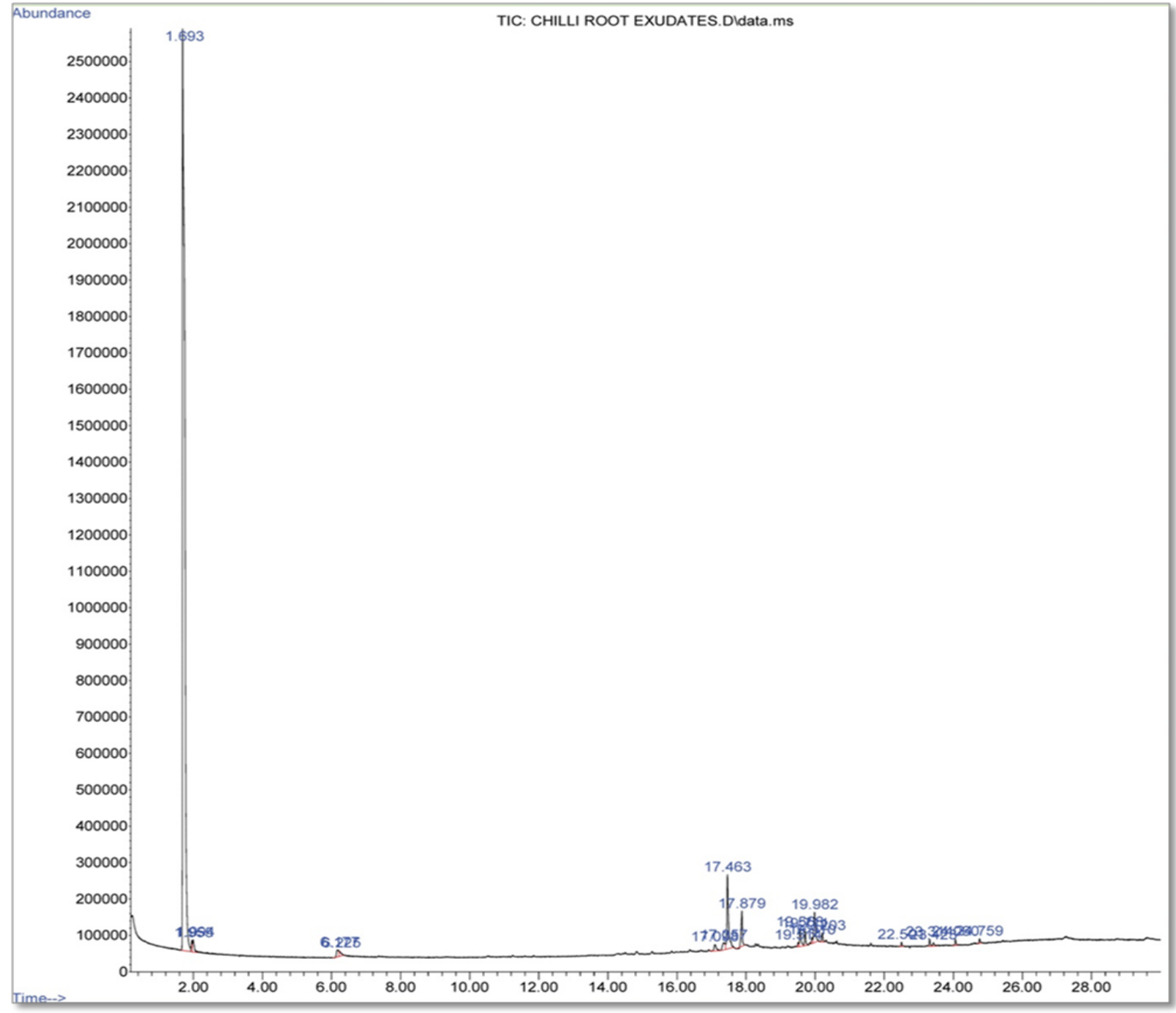

Fig. 1. GC-MS chromatogram for untreated 15 days old chilli seedling root exudates.

Table 1. Volatile compounds identified from root exudates of untreated chilli seedling 15 days after sowing.

\begin{tabular}{|c|c|c|c|c|c|c|}
\hline $\begin{array}{c}\text { Peak } \\
\text { No }\end{array}$ & $\begin{array}{l}\text { Reten- } \\
\text { tion time } \\
\text { (min) }\end{array}$ & $\begin{array}{c}\text { Peak } \\
\text { area } \\
(\%)\end{array}$ & Name of compound & $\begin{array}{c}\text { Nature of com- } \\
\text { pound }\end{array}$ & Structure & $\begin{array}{c}\text { Activity of com- } \\
\text { pound }\end{array}$ \\
\hline 1 & 1.695 & 91.98 & Hydroxylamine & Amine & $\mathrm{H}$ & $\begin{array}{l}\text { Antioxidants } \\
\text { Promote-seed germi- } \\
\text { nation }\end{array}$ \\
\hline 2 & 1.979 & 3.47 & $\begin{array}{l}\text { Dimethoxydime- } \\
\text { thylsilane }\end{array}$ & Ether & 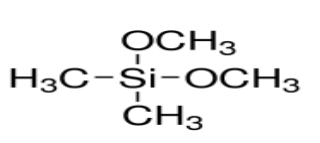 & $\begin{array}{l}\text { Precursor- } \\
\text { silicone polymer } \\
\text { polydimethylsilox- }\end{array}$ \\
\hline 3 & 17.467 & 1.02 & $\begin{array}{l}\text { Hexadecanoic acid, } \\
15 \text {-methyl, methyl } \\
\text { ester }\end{array}$ & Ester & & $\begin{array}{l}\text { Antioxidant, nemati- } \\
\text { cide, 5-alpha reduc- } \\
\text { tase inhibitor }\end{array}$ \\
\hline 4 & 19.574 & 0.86 & $\begin{array}{l}\text { Propanamide, 2- } \\
\text { methyl }\end{array}$ & Amide & & $\begin{array}{l}\text { Root growth modu- } \\
\text { lation }\end{array}$ \\
\hline 5 & 29.428 & 2.68 & Phenylephrine & $\begin{array}{l}\text { Phenethyla- } \\
\text { mines }\end{array}$ & & Antibacterial \\
\hline
\end{tabular}




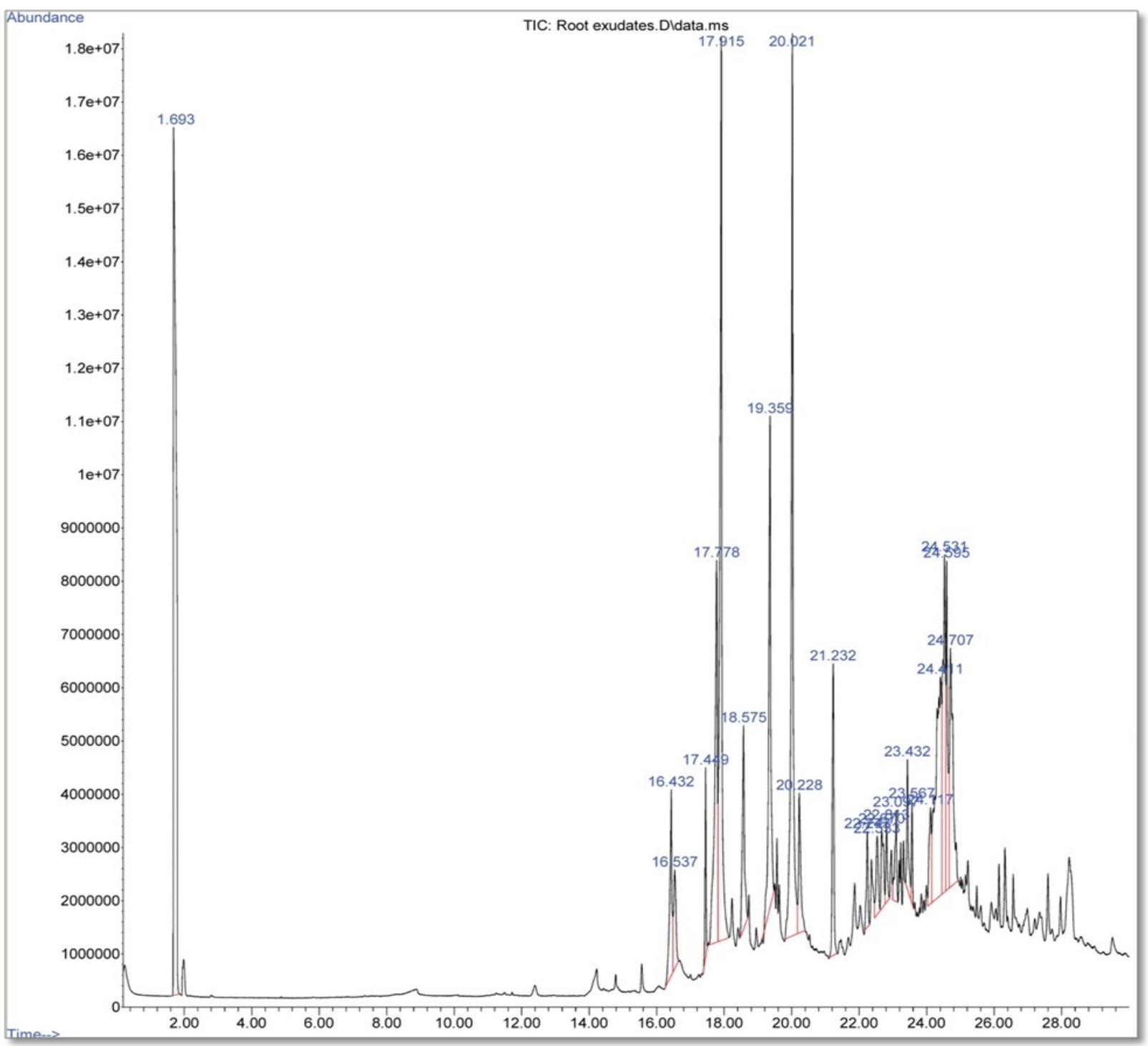

Fig. 2. GC-MS chromatogram for $6 \%$ Bacillus amyloliquefaciens VB7 and polymer coating treated 15 days old chilli seedling root exudates.

ronment by affecting non-target organisms. The fungal membrane has the fundamental role of maintaining cell order and integrity and hence antifungal treatment mostly target the fungal membrane (Avis, 2007). Avis and Belanger (2001) determined the general mechanism which antifungal fatty acids directly interacts with the fungal cell membrane. The antifungal fatty acids naturally insert themselves into the lipid bi-layer of the fungal membranes and physically disturb the membrane, resulting in increased fluidity of the membrane. These elevations in membrane fluidity will cause a generalized disorganization of the cell membrane that leads to conformational changes in membrane proteins, the release of intracellular components, cytoplasmic disorder and eventually cell disintegration.

Identification of volatile compounds in the root exudates of 30 days old chilli seedlings: The compounds identified in root exudates of nontreated seedlings was shown in Fig. 3 and Table 3. Among the identified compounds 1.3 per cent had antimicrobial activity. The major chemical constituents were hydroxylamine with peak area $(95.35 \%)$ and dimethoxydimethylsilane $(1.59 \%)$.

Twenty chemical constituents (Fig.4 and Table 4) have been identified from biopriming with 6 per cent $B$. amyloliquefaciens VB7 and polymer coating. Among the identified compounds 8.8 per cent had antimicrobial activity. The major chemical constituents with maximum peak area percentage in bacterized were identified as hydroxylamine (84.04\%), hexadecanoic acid15 methyl- methyl ester (4.84\%), n-decanoic acid (1.83\%), acetamide, 2,2,2-trifluoro (2.02\%).

The common compounds identified in treated and nontreated 30 day seedlings root exudates were hydroxylamine, dimethoxydimethylsilane, 2-heptanamine-5- 


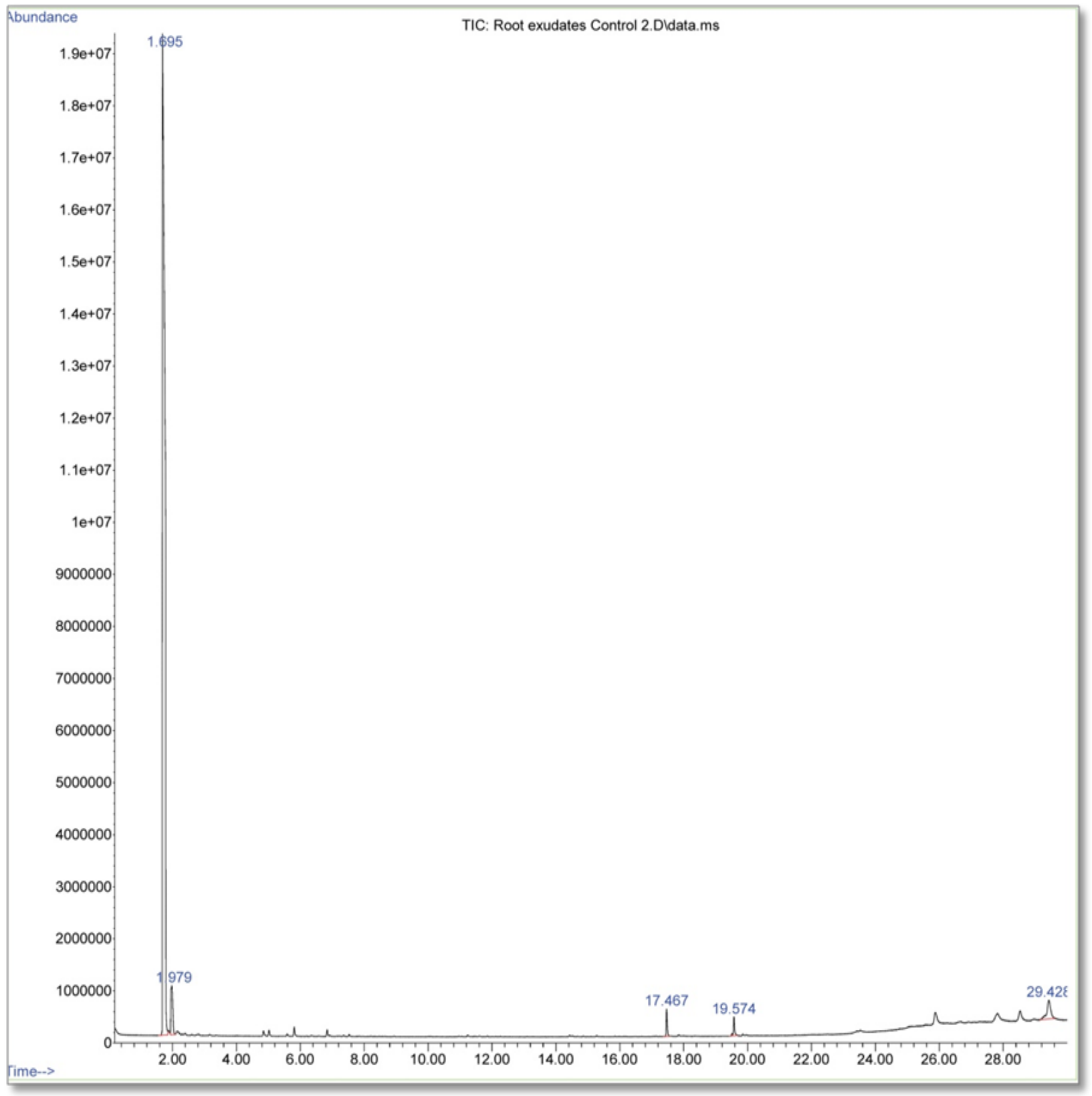

Fig. 3. GC-MS chromatogram for untreated 30 days old chilli seedling root exudates.

methyl, acetamide, 2,2,2-trifluoro, methylpent-4enylamine, hexadecanoic acid-15 methyl-methyl ester, phenylephrine, cyclobutanol, benzeneethanamine-4methoxy-alpha-methyl.

In this study, root exudates of 15 days old chilli seedling released more volatile compounds than the 30 days old chilli seedling. This result was closely agreeable with the reports of Rovira (1956). More amino acids and sugars were exuded during the first 10 days of growth than during the second 10 days in peas and oats. Vancura and Hovadik (1965) found that 3- pyrazolylalanine was present in the root exudates of cucumber only at the early stage.
The root exudations of volatile compounds are greatly influenced by root age of seedlings (Shukla et al., 2011). Microorganisms may affect the permeability of root cells, metabolism of roots, absorption and excretion of certain compounds in root exudates. It was reported that filtrates of cultures of some bacteria and fungi and also some antibiotics, increased the exudation by oat roots (Blaylock et al., 1997). Some other plant biotic factors like developmental status, shoot herbivory, photosynthesis, supply of carbon from shoot to root, evaporation, transpiration, nutrient deficiency, root architecture, cytosolic concentration, membrane permeability, membrane electrochemical potential, 
S. Sathya et al. / J. Appl. \& Nat. Sci. 8 (4): 2141-2154 (2016)

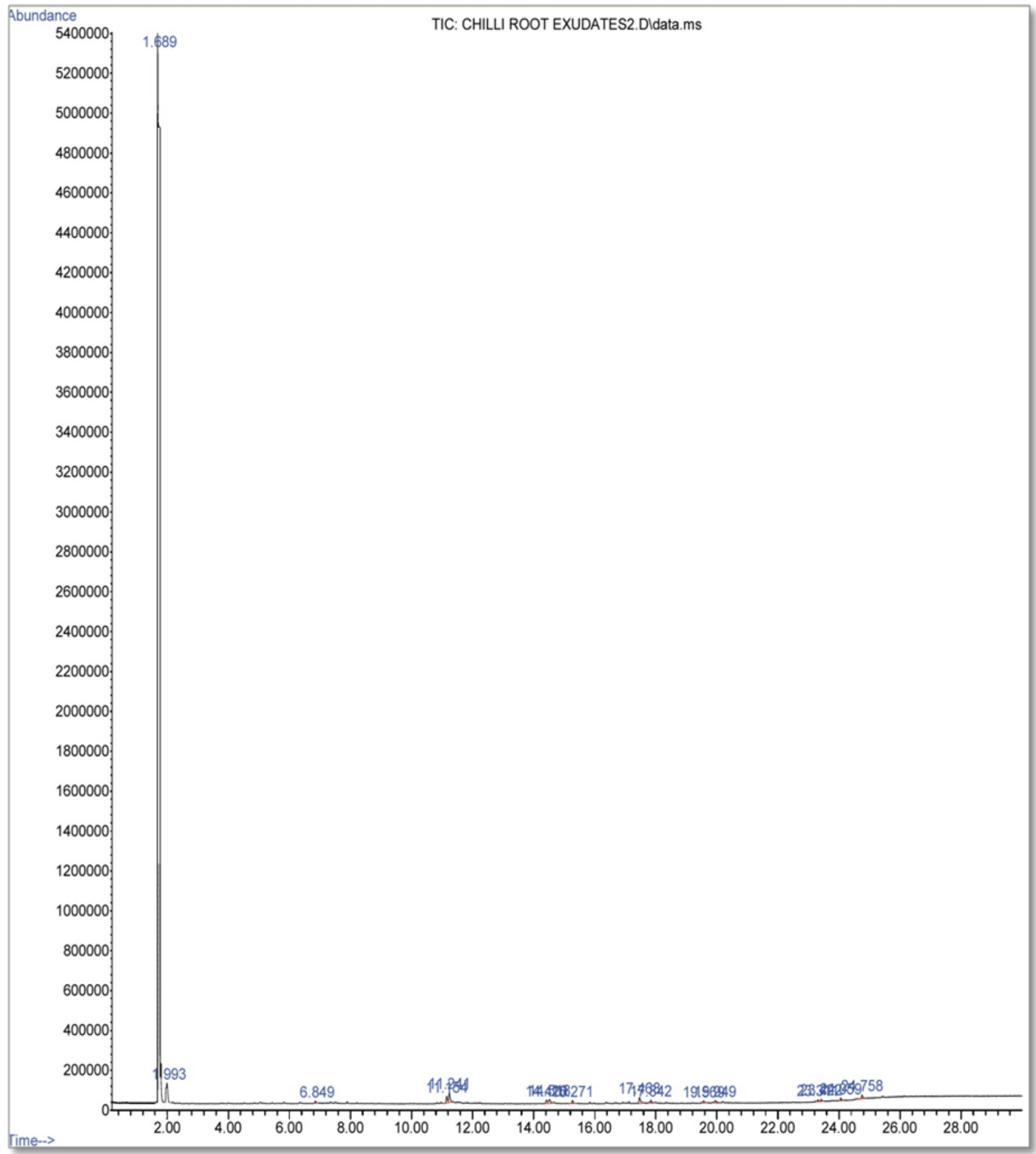

Fig. 4. GC-MS chromatogram for $6 \%$ Bacillus amyloliquefaciens VB7 and polymer coating treated 30 days old seedling root exudates.

release of microbial signal, allelochemical release, nodulation and some soil biotic factors are also influenced by the root exudation (Shukla et al., 2011).

Our results revealed that volatiles can have an effect on secondary metabolites production by $B$. amyloliquefaciens. When exposed to volatiles emitted by Collimonas pratensis, Pseudomonas fluorescens produced secondary metabolites that had inhibiting activity against a Gram positive bacterium and a fungus but not against the Gram negative volatile producer. It is plausible that the volatiles served as energy sources and/or signal inducing secondary metabolite production. The volatile triggered antibiotic production in $P$. fluorescens could point a strategy to combine movement (chemotaxis and motility genes) with increasing competitive strength (antibiotics) to invade in to the nutrient providing rhizosphere zone. It is known that bacterial volatiles can have antimicrobial activity and inhibit 
Table 2. Volatile compounds identified from root exudates of chilli seedling bioprimed with $6 \%$ Bacillus amyloliquefaciens and polymer coating after 15 days sowing.

\begin{tabular}{|c|c|c|c|c|c|c|}
\hline $\begin{array}{l}\text { Peak } \\
\text { No }\end{array}$ & $\begin{array}{l}\text { Retention } \\
\text { time(min) }\end{array}$ & $\begin{array}{l}\text { Peak } \\
\text { area } \\
(\%)\end{array}$ & $\begin{array}{l}\text { Name of com- } \\
\text { pound }\end{array}$ & $\begin{array}{l}\text { Nature of } \\
\text { compound }\end{array}$ & Structure & $\begin{array}{l}\text { Activity of com- } \\
\text { pound }\end{array}$ \\
\hline 1 & 1.693 & 10.13 & Hydroxylamine & Amine & $\mathbf{H}$ & $\begin{array}{l}\text { Antioxidants } \\
\text { Promote-seed germi- } \\
\text { nation }\end{array}$ \\
\hline 2 & 1.982 & 0.35 & $\begin{array}{l}\text { Dimethoxydime- } \\
\text { thyl silane }\end{array}$ & Ether & & $\begin{array}{l}\text { Precursor- } \\
\text { silicone polymer } \\
\text { polydimethylsiloxane }\end{array}$ \\
\hline 3 & 12.393 & 0.14 & $\begin{array}{l}\text { Hexadecanoic } \\
\text { acid, 15-methyl-, } \\
\text { methyl ester }\end{array}$ & Ester & & $\begin{array}{l}\text { Antioxidant, nemati- } \\
\text { cide, 5-alpha reduc- } \\
\text { tase inhibitor }\end{array}$ \\
\hline 4 & 14.223 & 0.42 & $\begin{array}{l}\text { Imidodicarbonic } \\
\text { diamide, } \mathrm{N} \text {-formyl }\end{array}$ & Amide & & Antimicrobial \\
\hline & & & & & & \\
\hline 5 & 14.787 & 0.15 & $\begin{array}{l}\text { Methyl 8-methyl- } \\
\text { decanoate }\end{array}$ & Ester & & Antifungal \\
\hline 6 & 15.560 & 0.20 & $\begin{array}{l}\text { Methyl 8-methyl- } \\
\text { nonanoate }\end{array}$ & Ester & & Antifungal \\
\hline 7 & 16.432 & 1.67 & Xylose & Sugar & & $\begin{array}{l}\text { Antibacterial, anti- } \\
\text { fungal, } \\
\text { Precursor-synthetic } \\
\text { polymers }\end{array}$ \\
\hline 8 & 16.537 & 1.02 & $\begin{array}{l}\text { Cyclopropane, } \\
\text { nonyl }\end{array}$ & $\begin{array}{l}\text { Cycloal- } \\
\text { kane }\end{array}$ & & $\begin{array}{l}\text { Antifungal-Pythium } \\
\text { spp. }\end{array}$ \\
\hline 9 & 17.449 & 1.07 & $\begin{array}{l}\text { Decanoic acid, } \\
\text { methyl ester }\end{array}$ & Ester & & Antifungal \\
\hline 10 & 17.532 & 0.21 & $\begin{array}{l}11,14- \\
\text { Eicosadienoic } \\
\text { acid, methyl ester }\end{array}$ & Ester & & Antibacterial \\
\hline 11 & 17.778 & 5.26 & cis-Undec-4-enal & Aldehyde & & Antimicrobial \\
\hline 12 & 17.915 & 9.24 & n-Decanoic acid & Fatty acid & & Antifungal \\
\hline 13 & 18.234 & 0.94 & 1-Eicosanol & $\begin{array}{l}\text { Fatty alco- } \\
\text { hol }\end{array}$ & & $\begin{array}{l}\text { Antifungal, } \\
\text { Antioxidant }\end{array}$ \\
\hline 14 & 18.415 & 0.42 & $\begin{array}{l}\text { 1,15- } \\
\text { Pentadecanediol }\end{array}$ & $\begin{array}{l}\text { Fatty alco- } \\
\text { hol }\end{array}$ & & $\begin{array}{l}\text { Antifungal, antibac- } \\
\text { terial Contd..... }\end{array}$ \\
\hline
\end{tabular}




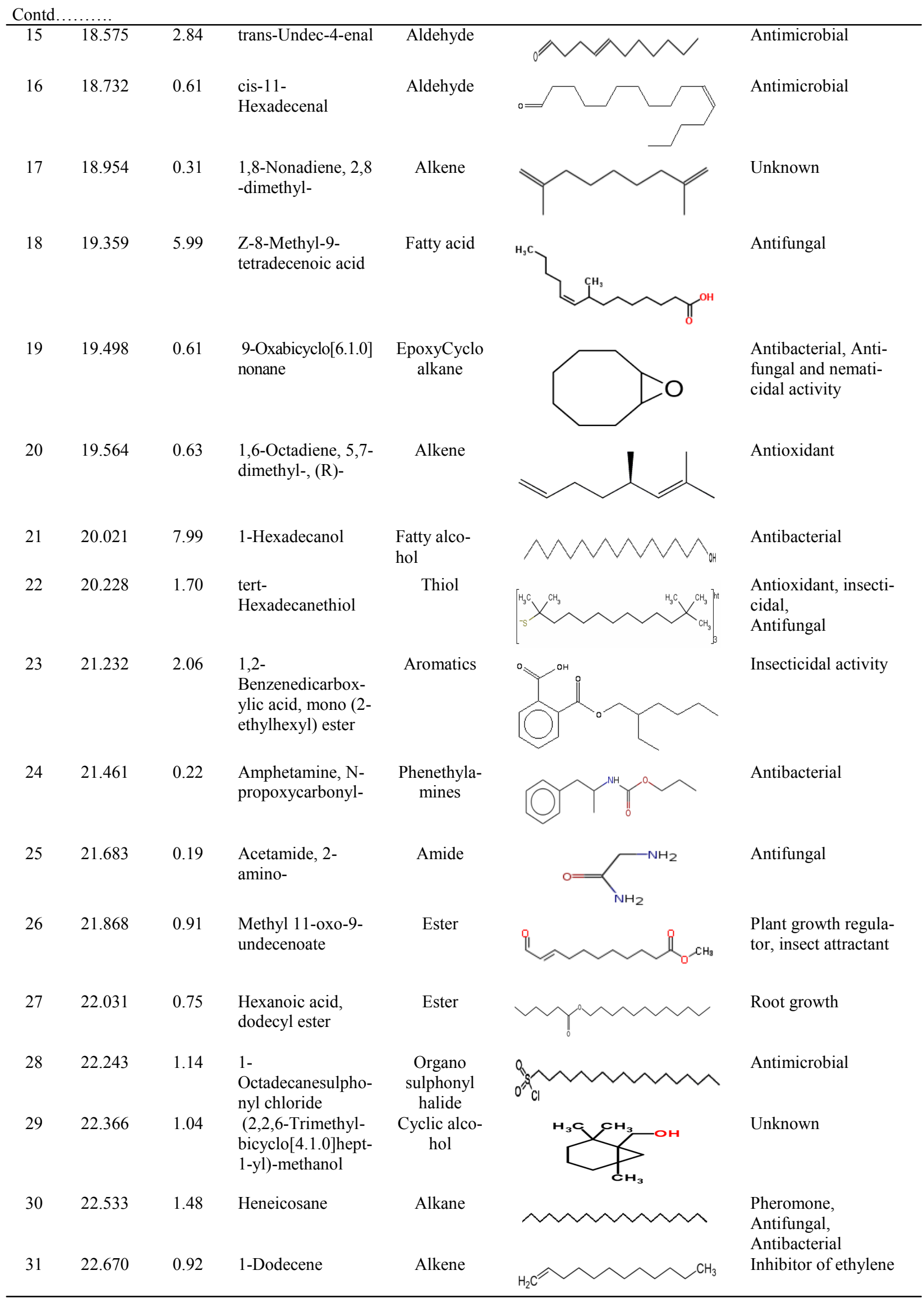


Contd.....

\begin{tabular}{|c|c|c|c|c|c|c|}
\hline 32 & 22.714 & 0.65 & $\begin{array}{l}\text { 4- } \\
\text { Heptafluorobutyr- } \\
\text { yloxyhexadecane }\end{array}$ & $\begin{array}{l}\text { Halogenat- } \\
\text { ed alkane }\end{array}$ & 0 & Antibacterial \\
\hline 33 & 22.813 & 1.28 & $\begin{array}{l}\text { Heptane, 3-ethyl- } \\
\text { 2-methyl }\end{array}$ & Alkane & & $\begin{array}{l}\text { To induce shortening } \\
\text { and thickening of the } \\
\text { stem in cereals and } \\
\text { other crop plants in- } \\
\text { creasing the yields or } \\
\text { setting of fruit }\end{array}$ \\
\hline 34 & 22.955 & 0.88 & $\begin{array}{l}3-[\mathrm{N}-\text { Aziridyl }] \\
\text { propionyl hydra- } \\
\text { zide }\end{array}$ & Hydrazide & & Antimicrobial \\
\hline 35 & 23.097 & 1.63 & Cyclodecanone & $\begin{array}{l}\text { Alicyclic } \\
\text { Ketone }\end{array}$ & & $\begin{array}{l}\text { Insecticidal } \\
\text { Fungicidal }\end{array}$ \\
\hline 36 & 23.234 & 0.56 & $\begin{array}{l}\text { 2-Piperidinone, 6- } \\
\text { methyl }\end{array}$ & $\begin{array}{l}\text { Cyclic am- } \\
\quad \text { ide }\end{array}$ & & Antifungal \\
\hline 37 & 23.315 & 0.85 & $\begin{array}{l}\mathrm{N}- \\
\text { Acryloylsarcosine } \\
\text { methyl ester }\end{array}$ & Ester & & Unknown \\
\hline 38 & 23.432 & 1.82 & Decane, 1-fluoro- & $\begin{array}{l}\text { Halogenat- } \\
\text { ed alkane }\end{array}$ & & $\begin{array}{l}\text { Intra and inter-plant } \\
\text { communication } \\
\text { Attraction or repulsion } \\
\text { of parasites }\end{array}$ \\
\hline 39 & 23.567 & 0.90 & $\begin{array}{l}\text { Octadecane, 1- } \\
\text { chloro- }\end{array}$ & $\begin{array}{l}\text { Halogenat- } \\
\text { ed alkane }\end{array}$ & $v$ & Antifungal \\
\hline 40 & 23.790 & 0.21 & $\begin{array}{l}\text { Ethanamine, 2- } \\
\text { phenoxy }\end{array}$ & Amine & & $\begin{array}{l}\text { Root growth and devel- } \\
\text { opment }\end{array}$ \\
\hline 41 & 23.843 & 0.35 & $\begin{array}{l}\text { Cyclooctene, 1,2- } \\
\text { dimethyl- }\end{array}$ & Cycloalkene & & $\begin{array}{l}\text { Insecticidal } \\
\text { (shoot fly resistance) }\end{array}$ \\
\hline 42 & 23.997 & 0.41 & $\begin{array}{l}\text { 3- } \\
\text { Trifluoroacetoxy- } \\
\text { pentadecane }\end{array}$ & $\begin{array}{l}\text { Halogenat- } \\
\text { ed alkane }\end{array}$ & $\mathrm{CH}_{3}$ & Antimicrobial \\
\hline 43 & 24.117 & 1.42 & $\begin{array}{l}\text { 8-Hexadecenal, } \\
\text { 14-methyl-, (Z)- }\end{array}$ & Aldehyde & 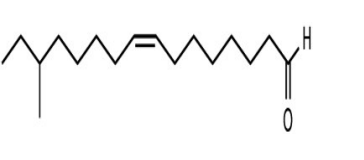 & $\begin{array}{l}\text { Pesticide-Pheromone } \\
\text { Khapra beetle } \\
\text { (BallionWarehouse } \\
\text { beetle) }\end{array}$ \\
\hline 44 & 24.312 & 3.35 & 9-Octadecenal & $\begin{array}{c}\text { Fatty } \\
\text { aldehyde }\end{array}$ & 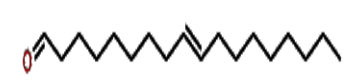 & Antifungal \\
\hline 45 & 24.411 & 1.91 & $\begin{array}{l}\text { 18-Nonadecen-1- } \\
\text { ol }\end{array}$ & $\begin{array}{l}\text { Fatty } \\
\text { alcohol }\end{array}$ & ${ }_{M} M M M^{\circ}$ & Antibacterial \\
\hline 46 & 24.531 & 4.35 & 13-Tetradecenal & Aldehyde & & $\begin{array}{l}\text { Antimicrobial, } \\
\text { Insecticidal }\end{array}$ \\
\hline 47 & 24.595 & 2.93 & $\begin{array}{l}\text { Tetrapentacon- } \\
\text { tane, } 1,54- \\
\text { dibromo- }\end{array}$ & $\begin{array}{l}\text { Halogen- } \\
\text { atedalkane }\end{array}$ & $m$ & $\begin{array}{l}\text { Antimicrobial and } \\
\text { antifungal }\end{array}$ \\
\hline
\end{tabular}




\begin{tabular}{|c|c|c|c|c|c|c|}
\hline 47 & 24.595 & 2.93 & $\begin{array}{l}\text { Tetrapentacontane, } \\
\text { 1,54-dibromo- }\end{array}$ & $\begin{array}{l}\text { Halogen- } \\
\text { atedalkane }\end{array}$ & $m$ & $\begin{array}{l}\text { Antimicrobial and } \\
\text { antifungal }\end{array}$ \\
\hline 48 & 24.707 & 4.46 & 13-Octadecenal, (Z)- & Aldehyde & & $\begin{array}{l}\text { Pesticide- } \\
\text { Pheromone }\end{array}$ \\
\hline 49 & 25.063 & 0.34 & $\begin{array}{l}\text { Acetic acid, trichloro } \\
- \text {, nonyl ester }\end{array}$ & Ester & & Pesticide \\
\hline 50 & 25.152 & 0.50 & 1-Hexacosanol & $\begin{array}{l}\text { Fatty alco- } \\
\text { hol }\end{array}$ & & Antifungal \\
\hline 51 & 25.223 & 0.62 & $\begin{array}{l}\text { Hexadecane, 1- } \\
\text { bromo- }\end{array}$ & $\begin{array}{l}\text { Halogenated } \\
\text { alkane }\end{array}$ & & Antifungal \\
\hline 52 & 25.485 & 0.26 & 1-Bromodocosane & $\begin{array}{l}\text { Halogenated } \\
\text { alkane }\end{array}$ & & Antifungal \\
\hline 53 & 25.606 & 0.17 & $\begin{array}{l}\text { Propanenitrile, 3- } \\
\text { amino-2,3-di } \\
\text { (hydroxymino)- }\end{array}$ & $\begin{array}{l}\text { Aliphatic } \\
\text { nitrile }\end{array}$ & & Unknown \\
\hline 54 & 25.916 & 0.52 & Heptanal & Aldehyde & & Lipid oxidation \\
\hline 55 & 26.050 & 0.20 & Undecanal & Aldehyde & & Antibacterial \\
\hline 56 & 26.145 & 0.38 & Piperazine, 2-methyl- & Piperazine & & $\begin{array}{l}\text { Antifungal antibac- } \\
\text { terial and Plant } \\
\text { growth regulation }\end{array}$ \\
\hline 57 & 26.321 & 0.60 & $\begin{array}{l}\text { 7-Oxabicyclo[4.1.0] } \\
\text { heptane }\end{array}$ & $\begin{array}{l}\text { Epoxycyclo } \\
\text { alkane }\end{array}$ & & $\begin{array}{l}\text { Antibacterial } \\
\text { Antifungal } \\
\text { Nematicidal }\end{array}$ \\
\hline 58 & 26.401 & 0.09 & $\begin{array}{l}\text { Oxalic acid, allyl } \\
\text { hexadecyl ester }\end{array}$ & Fatty acid & & Antimicrobial \\
\hline 59 & 26.567 & 0.49 & $\begin{array}{l}\text { 3-Hydroxy-N,N- } \\
\text { dimethylpropana- } \\
\text { mide }\end{array}$ & Amide & $\mathrm{CH}_{3}$ & Unknown \\
\hline 60 & 26.982 & 0.57 & $\begin{array}{l}\text { Urea, (hexahydro-6- } \\
\text { methyl-2-oxo-4- } \\
\text { pyrimidinyl)- }\end{array}$ & Amide & & $\begin{array}{l}\text { Plant growth regu- } \\
\text { lation }\end{array}$ \\
\hline 61 & 27.344 & 0.50 & $\begin{array}{l}\text { Bicyclo[2.1.1] hexane } \\
\text {-1-carboxylic acid, } \\
\text { 5,5-dimethyl- }\end{array}$ & $\begin{array}{l}\text { Carboxylic } \\
\text { acid }\end{array}$ & & Antifungal \\
\hline 62 & 27.596 & 0.50 & $\begin{array}{l}\text { Sarcosine, N-valeryl- } \\
\text {, ethyl ester }\end{array}$ & Ester & & $\begin{array}{l}\text { Increase plant bio- } \\
\text { mass }\end{array}$ \\
\hline 63 & 27.967 & 0.38 & $\begin{array}{l}\text { 7-Oxabicyclo[4.1.0] } \\
\text { heptane, 1-methyl-4-(2 } \\
\text {-methyloxiranyl) }\end{array}$ & $\begin{array}{l}\text { Epoxy cyclo } \\
\text { alkane }\end{array}$ & & $\begin{array}{l}\text { Antibacterial } \\
\text { Antifungal } \\
\text { Nematicidal }\end{array}$ \\
\hline 64 & 28.223 & 1.78 & $\begin{array}{l}\text { 2,6,6-Trimethyl- } \\
\text { bicyclo[3.1.1]hept-3- } \\
\text { ylamine }\end{array}$ & $\begin{array}{l}\text { Bicyclo } \\
\text { amine }\end{array}$ & & Insecticidal \\
\hline 65 & 29.505 & 0.26 & $\begin{array}{l}\text { Benzenemethanol, } \\
\text { alpha. } \\
\text { [(methylamino) me- } \\
\text { thyl]- }\end{array}$ & Aromatic & & Unknown \\
\hline
\end{tabular}


S. Sathya et al. / J. Appl. \& Nat. Sci. 8 (4): 2141-2154 (2016)

Table 3. Volatile compounds identified from root exudates of untreated chilli seedling 30 days after sowing.

\begin{tabular}{|c|c|c|c|c|c|c|}
\hline $\begin{array}{c}\text { Pea } \\
\mathbf{k} \\
\text { No }\end{array}$ & $\begin{array}{c}\text { Reten- } \\
\text { tion } \\
\text { time } \\
\text { (min) }\end{array}$ & $\begin{array}{l}\text { Peak } \\
\text { area } \\
(\%)\end{array}$ & Name of compound & $\begin{array}{l}\text { Nature of } \\
\text { compound }\end{array}$ & Structure & Activity of compound \\
\hline 1 & 1.689 & 95.35 & Hydroxylamine & Amine & $\dot{H}$ & $\begin{array}{l}\text { Antioxidants } \\
\text { Promote-seed germination }\end{array}$ \\
\hline 2 & 1.993 & 1.59 & $\begin{array}{l}\text { Dimethoxydime- } \\
\text { thylsilane }\end{array}$ & Ether & $\begin{array}{c}\mathrm{OCH}_{3} \\
\stackrel{1}{\mathrm{H}} \mathrm{C}-\mathrm{OCH}_{3} \\
\stackrel{\mathrm{S}}{\mathrm{I}}-\mathrm{OCH}_{3} \\
\mathrm{CH}_{3}\end{array}$ & $\begin{array}{l}\text { Precur- } \\
\text { sorsilicone polymerpolydi } \\
\text { methylsiloxane }\end{array}$ \\
\hline 3 & 6.849 & 0.10 & $\begin{array}{l}\text { Acetic acid, } \\
\text { [(aminocarbonyl) } \\
\text { amino]oxo }\end{array}$ & Acid & & Disease resistance \\
\hline 4 & 11.154 & 0.38 & $\begin{array}{l}\text { 2-Heptanamine, 5- } \\
\text { methyl }\end{array}$ & Amine & & Antimicrobial \\
\hline 5 & 11.241 & 0.56 & 1-Hexadecanol & $\frac{\text { Fatty alco- }}{\text { hol }}$ & & Antibacterial \\
\hline 6 & 14.420 & 0.20 & $\begin{array}{l}\text { Acetamide, 2,2- } \\
\text { dichloro }\end{array}$ & Amide & & Root development \\
\hline 7 & 14.518 & 0.34 & $\begin{array}{l}\text { Benzeneethanamine, } \mathrm{N} \\
\text {-methyl }\end{array}$ & Amine & & $\begin{array}{l}\text { Plant resistance, } \\
\text { Antioxidant }\end{array}$ \\
\hline 8 & 15.271 & 0.16 & $\begin{array}{l}\text { Methylpent-4- } \\
\text { enylamine }\end{array}$ & Amine & & Antibacterial \\
\hline 9 & 17.468 & 0.40 & $\begin{array}{l}\text { Hexadecanoic acid, 15- } \\
\text { methyl-, methyl ester }\end{array}$ & Ester & & $\begin{array}{l}\text { Antioxidant, nematicide, } \\
5 \text {-alpha reductase inhibi- } \\
\text { tor }\end{array}$ \\
\hline 10 & 17.842 & 0.16 & Phenylephrine & $\begin{array}{l}\text { Phenethyla } \\
\text {-mines }\end{array}$ & & Antibacterial \\
\hline 11 & 19.569 & 0.12 & Cyclobutanol & Alcohol & & $\begin{array}{l}\text { Plant resistance, } \\
\text { Antioxidant }\end{array}$ \\
\hline 12 & 19.949 & 0.21 & $\begin{array}{l}\text { 1-Methyl-2- } \\
\text { phenoxyethylamine }\end{array}$ & Amine & & Plant growth regulation \\
\hline 13 & 23.311 & 0.08 & $\begin{array}{l}\text { 2-Thiophenecarboxylic } \\
\text { acid, 5-(1,1- } \\
\text { dimethylethoxy)- }\end{array}$ & $\begin{array}{l}\text { Organosul- } \\
\text { fur }\end{array}$ & & Plant growth regulation \\
\hline 14 & 24.059 & 0.11 & $\begin{array}{l}\text { N-Methyl-2-phenyl-1- } \\
\text { propanamine }\end{array}$ & Amine & & $\begin{array}{l}\text { Plant resistance, } \\
\text { Antioxidant }\end{array}$ \\
\hline 15 & 24.758 & 0.23 & $\begin{array}{l}\text { Benzeneethanamine, 4- } \\
\text { methoxy-alpha-methyl }\end{array}$ & Amine & & $\begin{array}{l}\text { Plant resistance, } \\
\text { Antioxidant }\end{array}$ \\
\hline
\end{tabular}


Table 4. Volatile compounds identified from root exudates of chilli seedling bioprimed with $6 \%$ Bacillus amyloliquefaciens and polymer coating after 30 days sowing.

\begin{tabular}{|c|c|c|c|c|c|c|}
\hline $\begin{array}{c}\text { Pea } \\
\mathbf{k} \\
\text { No }\end{array}$ & $\begin{array}{l}\text { Reten- } \\
\text { tion } \\
\text { time } \\
(\mathrm{min})\end{array}$ & $\begin{array}{l}\text { Peak } \\
\text { area } \\
(\%)\end{array}$ & $\begin{array}{l}\text { Name of com- } \\
\text { pound }\end{array}$ & $\begin{array}{l}\text { Nature of } \\
\text { compound }\end{array}$ & Structure & Activity of compound \\
\hline 1 & 1.693 & 84.04 & Hydroxylamine & Amine & H & $\begin{array}{l}\text { Antioxidants } \\
\text { Promote-seed germination }\end{array}$ \\
\hline 2 & 1.955 & 0.37 & $\begin{array}{l}1,2,3,4- \\
\text { Butanetetrol, } \\
{\left[\mathrm{S}-\left(\mathrm{R}^{*}, \mathrm{R}^{*}\right)\right]}\end{array}$ & $\begin{array}{l}\text { Alcohol } \\
\text { (Sugar alco- } \\
\text { hol) }\end{array}$ & & $\begin{array}{l}\text { Antimicrobial, } \\
\text { Plant growth regulation }\end{array}$ \\
\hline 3 & 1.994 & 0.78 & $\begin{array}{l}\text { Dimethoxydime- } \\
\text { thylsilane }\end{array}$ & Ether & & $\begin{array}{l}\text { Precursor-silicone polymer } \\
\text { polydimethylsiloxane }\end{array}$ \\
\hline 4 & 6.177 & 0.56 & $\begin{array}{l}\text { 2-Heptanamine, } 5 \\
\text {-methyl }\end{array}$ & Amine & & Antimicrobial \\
\hline 5 & 6.225 & 0.42 & $\begin{array}{l}\text { Cyclohexan- } \\
\text { 1,4,5-triol-3-one } \\
\text {-1-carboxylic } \\
\text { acid }\end{array}$ & Ester & & Antibacterial \\
\hline 6 & 17.098 & 0.62 & $\begin{array}{l}\text { 1,3-Dioxolane-4 } \\
\text {-methanol }\end{array}$ & Hemiacetal & & Root growth \\
\hline 7 & 17.357 & 0.78 & $\begin{array}{l}\text { 1-Pentanol, 4- } \\
\text { amino }\end{array}$ & $\begin{array}{l}\text { Amino } \\
\text { alcohol }\end{array}$ & & $\begin{array}{l}\text { Root growth, } \\
\text { Stress tolerance }\end{array}$ \\
\hline 8 & 17.463 & 4.84 & $\begin{array}{l}\text { Hexadecanoic } \\
\text { acid, 15-methyl, } \\
\text { methyl ester }\end{array}$ & Ester & & $\begin{array}{l}\text { Antioxidant, nematicide, 5- } \\
\text { alpha reductase inhibitor }\end{array}$ \\
\hline 9 & 17.879 & 1.83 & n-Decanoic acid & Fatty acid & & Antifungal \\
\hline 10 & 19.514 & 0.18 & Cyclobutanol & Alcohol & & $\begin{array}{l}\text { Plant resistance, } \\
\text { Antioxidant }\end{array}$ \\
\hline 11 & 19.568 & 1.02 & $\begin{array}{l}\text { p- } \\
\text { Hydroxy- } \\
\text { norephedrine }\end{array}$ & $\begin{array}{l}\text { Phenethyla- } \\
\text { mines }\end{array}$ & & Antibacterial \\
\hline
\end{tabular}


Contd........

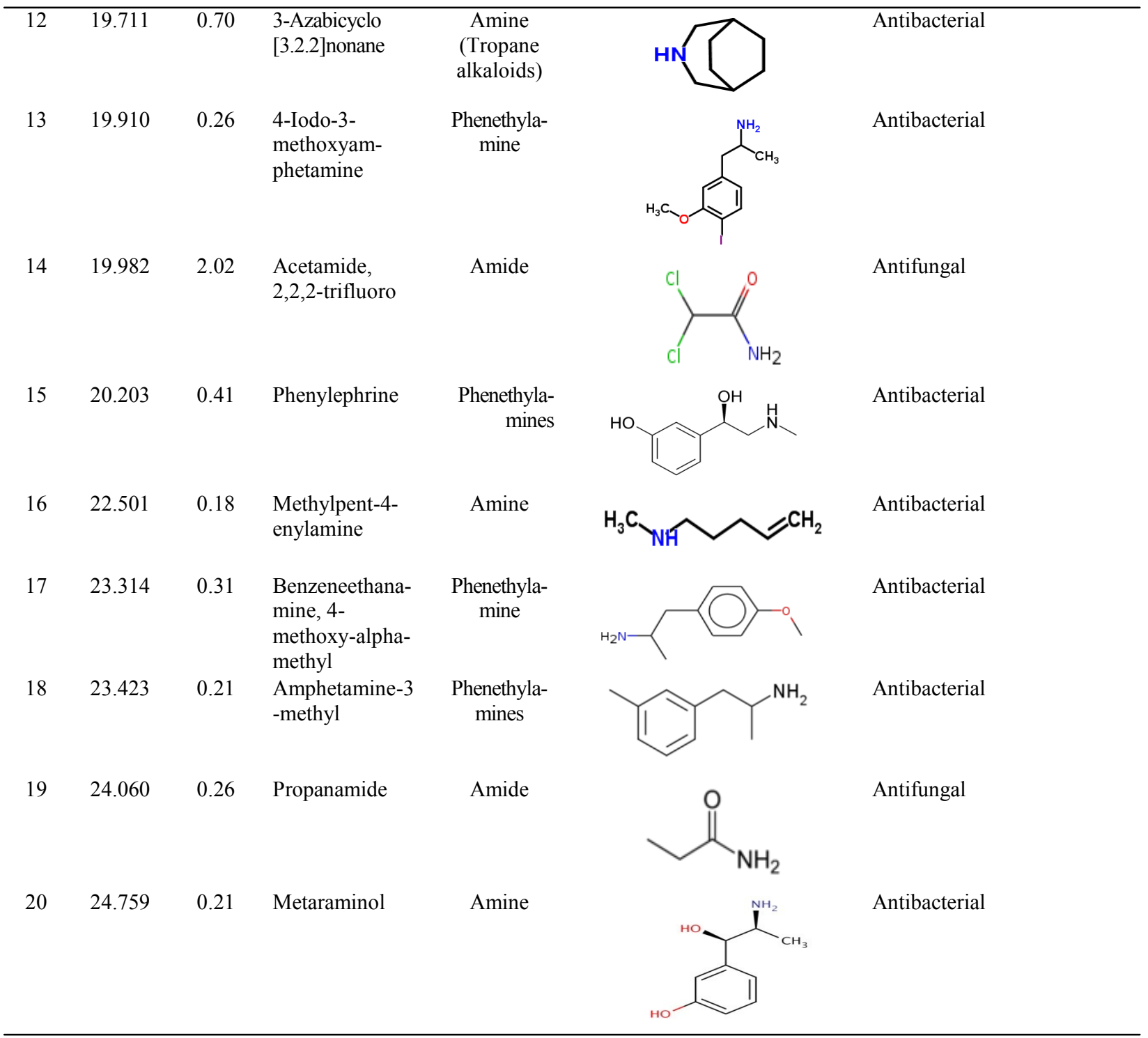

the growth of other microorganisms (Kai et al., 2007, 2009; Garbeva et al., 2014a, 2014b).

\section{Conclusion}

It was concluded that, the common compound identified in both 15 and 30 day old chilli seedling were, hydroxylamine, dimethoxydimethyl silane and hexadecanoic acid -15-methyl- methyl ester. The results on GCMS analysis revealed that root exudates collected from 15 and 30 day old bacterized seedlings with B. amyloliquefaciens VB7 and polymer coating released more number of volatile compounds than control. Between 15 and 30 day old seedlings, biopriming with $B$. amyloliquefaciens VB7 and polymer coated 15 day old seedling root exudates released more volatile compounds than 30 day old seedling. Majority of the compounds in bacterized seedlings had antimicrobial activity. Those compounds indicate their potential use for various diseases in traditional system.

\section{REFERENCES}

Archibold, D.D., Hamilton-Kemp, T.R., Barth, M.M. and Langlois, B.E. (1997). Identifying natural volatile compounds that control gray mold (Botrytis cinerea) during postharvest storage of strawberry, blackberry and grape. J. Agricultural and Food Chemistry, 45: 4032-4037

Avis, T.J. (2007). Antifungal compounds that target fungal membrane: Application in plant disease control. Can. J. Pl. Path., 29: 323-329

Avis, T.J. and Bélanger, R.R. (2001). Specificity and mode of action of the antifungal fatty acid cis-9-heptadecenoic acid produced by Pseudozyma flocculosa. Appl. Environ. Microbiol., 67: 956-960

Bais, H.P., Weir, T.L., Perry, L.G., Gilroy, S. and Vivanco, J.M. (2006). The role of root exudates in rhizosphere interactions with plants and other organisms. Annu. Rev. Pl. Biol., 57: 233-266

Blaylock, M.S.D.E., Dushenkv, S., Zakharavo, O., Gussman, 
C., Kapulnik, Y., Ensley, B. and Raskin, E. (1997). Enhanced accumulation of $\mathrm{Pb}$ in Indian mustard by soilapplied chelating agents. Environ. Sci. Technol., 31: 860 $-865$

Carballeira, N.M. (2008). New advances in fatty acids as antimalarial, antimycobacterial and antifungal agents A review. Progress in Lipid Research, 47:50-61

Dorlodot, S., Forster, B., Pages, L., Price, A., Tuberosa, R. and Draye, X. (2007). Root system architecture: Opportunities and constraints for genetic improvement of crops. Trends Plant Sci., 12: 474-481

Garbeva, P., Hordijk, C., Gerards, S. and DeBoer, W. (2014a). Volatile-mediated interactions between phylogenetically different soil bacteria. Frontiers in Microbiology, 5: 1-9

Garbeva, P., Hordijk, C., Gerards, S. and DeBoer, W. (2014b). Volatiles produced by the mycophagous soil bacterium Collimonas. FEMS Microbiol. Ecol., 87: 639649

Gibeaut, D.M., Hulett, J., Cramer, G.R. and Seemann, J.R. (1997). Maximal biomass of Arabidopsis thaliana using a simple, low maintenance hydroponic method and favourable environmental conditions. Plant Physiol., 115: 317-319

Hendricks, S.B. and Taylorson, R.B. (1974). Promotion of seed germination by nitrate, nitrite, hydroxylamine, and ammonium salts. Plant physiol., 54: 304-309

Heydecker, W. (1973). Germination of an idea: The priming of seeds. University of Nottingham School of Agriculture Rep., 74

Hoagland, D.R. and Arnon, D.I. (1950). The water culture method for growing plants without soil. California Agricultural Experimental Station Circular No. 347, pp. 132. University of California, Berkeley.

Kai, M., Effmert, U., Berg, G. and Piechulla, B. (2007).Volatiles of bacterial antagonists inhibit mycelial growth of the plant pathogen Rhizoctonia solani. Arch. Microbiol., 187: 351-360

Kai, M., Haustein, M., Molina, F., Petri, A., Scholz, B. and Piechulla, B. (2009). Bacterial volatiles and their action potential. Appl. Microbiol. Biotechnol, 81: 1001-1012

Liu, S., Weibin, R., Jing, L., Hua, X., Jingan, W., Yubao, G. and Jingguo, W. (2008). Biological control of phytopathogenic fungi by fatty acids. Mycopathologia, 66: 93-102

Mahadkar, S., Valvi, S. and Jadhav, V. (2013). Gas chromatography mass spectroscopic (GCMS) analysis of some bioactive compounds form five medicinally relevant wild edible plants. Asian J. Pharm. Clin. Res., 6(1): 136 $-139$

Nakkeeran, S., Kavitha, K., Chandrasekar, G., Renukadevi, P. and Fernando, W.G.D. (2006). Induction of plant defence compounds by Pseudomonas chlororaphis PA23 and Bacillus subtilis BSCBE4 in controlling damping-off of hot pepper caused by Pythium apha- nidermatum. Biocontrol Sci. and Technol., 16(4): 403416

Nguyen, C. (2003). Rhizodeposition of organic C by plants: Mechanisms and controls. Agronomy, 23: 375-396

Qun, H. Z., Junan, Z., HaoRu, T. and Zhi, H. (2012). Different vegetables crops in response to allelopathic of hot pepper root exudates. World Appl. Sci. J., 19 (9): 12891294

Rao, M.S.L., Kulkarni, S., Sagar, S.D. and Kulkarni, V.R. (2007). Biopriming induced changes in the activity of defence related enzymes for conferring resistance against Alternaria blight of sunflower. J. Pl. Dis. Sci., 2 (1): 14-17

Rovira, A.D. (1956). A study of the development of the root surface microflora during the initial stages. J. Appl. Bacteriol., 19:72-79

Selvamangai, G. and Anusha, B. (2012). GC-MS analysis of phytocomponents in the methanolic extract of Eupatorium triplinerve. Asian Pacific Journal of Tropical Biomedicine, 329-332

Shah Smith, D.A. and Burns, R.G. (1996). Biological control of damping off of sugar beet by Pseudomonas putida applied to seed pellets. Plant pathol., 45: 572-582

Shukla, K.V., Sharma, S., Singh, N.K., Singh, V., Tiwari, K. and Singh, S. (2011). Nature and role of root exudates: Efficacy in bioremediation. African J. Biotechnol., 10 (48): 9717-9724

Sowmini, R. (1961). Studies on Phycomycetes in agricultural soils with special reference to Pythiaceae. M.Sc. (Agri.) Thesis, University of Madras: 160

Taylor, T.W.J. and Baker, W. (1937). Sidgwich's organic chemistry of nitrogen. Oxford University Press, New York. pp. 166-169

Vanangamudi, K., Srimathi, P., Natarajan, N. and Bhaskaran, M. (2003). Current scenario of seed coating polymer. In: Proc. of ICAR short course on seed hardening and pelleting technologies for rainfed/ garden land ecosystems, New Delhi, pp. 80-100.

Vancura, V. and Hovadik, A. (1965). Composition of root exudates in the course of plant development. Plant Microb. Relat., pp. 21-25

Weller, D.M. (1984). Distribution of a take-all suppressive strain of Pseudomonas fluorescens on seminal roots of winter wheat. Appl. Environ. Microbiol., 48(4): 897-899

Weller, D.M. and Cook, R.J. (1983). Suppression of take-all of wheat by seed treatment with Pseudomonas fluorescent. Phytopathol., 73: 463-469

Whipps J.M. (1990). Carbon economy. In: The rhizosphere (ed. J.M. Lynch), pp. 59-97. JohnWiley \& Sons Ltd, Essex, UK

Zagade, S.N., Deshpande, G.D., Gawade, D.B., Atnoorkar, A.A. and Pawar, S.V. (2012). Biocontrol agents and fungicides for management of damping off in chilli. World J. Agric. Sci., 8 (6): 590-597 